\title{
Un criterio pratico di trasformazione fra diagrammi di resistività apparente ottenuti con differenti dispositivi
}

\author{
R. C'ASSINIS
}

Da lungo tempo si discute sulla più conreniente configurazione geometrica dello stendimento da adottare nei sondaggi elettrici verticali. Per varie ragioni, del resto ormai ben note, la maggiomanza degli operatori temale ad usare il dispositivo Schlumberger; in questo schema la distanza trat le sonde del potenziale resta fissa mentre rengono spostati gli elettrodi di corrente oppure, se la distanza tra le sonde viene variata, la misura viene ripetuta lasciando fissi gli elettrocli.

I cliagrammi teorici interpretativi vengono calcolati in nuagronianza per il dispositivo Schlumberger nella ipotesi che la distanza tra gli elettrodi potenziometrici sia infinitesima; il recchio metodo Wenner però, ì ancora molto diffuso; à assai utile, pertanto, poter aroperare i diagrammi ricarati con amberlue le ipotessi e, poichè il calcolo è assai laborioso, passare da man serie all'altra di curve merliante un procedimento semplice e sufficientemente approssimato.

A. Belluigi ( $\left.{ }^{1}\right)$ partendo dall'espressione generale della resistiviti per il polo singolo, trova la relazione segutente, che fornisce la differenza tra la resistività osservata $\left(o_{a}\right)$ mediante un lapporto $y=\frac{a}{a} \neq 0$ e quella $\left(g_{0}\right)$ che si otterrebbe con $y \rightarrow 0$ (dispositivo Schlumberger):

$\varrho_{a}-o_{n}=\frac{1-y^{2}}{2 a} \int_{-a}^{+a} \frac{\varrho(d+x)-\varrho(d)}{\left(1+\frac{x}{d}\right)^{2}}$

dove $d$ è la semidistanza tra gli elettrodi di corrente e $a$ è la semidistanza tra le sonde ilel potenziamento (fig. 1).
Lal correzione può essere ricarata direttamente dalla [1] alcolando l'integlale per via grafica; questo procedimento è ovviamente troppo laborioso ed oceorre pereio cercare un metodo piì rapido.

Per il calcolo approssimato conviene sviluppare in serie la funzione integrancla. Per

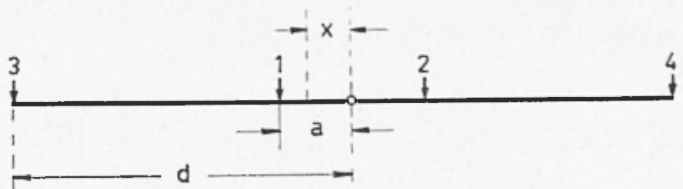

1-2: sonde del potenziale

3-4: elettrodi di corrente

Fig. I

lo sviluppo di o a limiteremo al termine di terzo grado, poichè ì praticamente impossibile calcolare le derivate superiori alla seconda, a l'ultinno termine ci servirà solo a nostrare (he in generale ì abbastanza piecolo da potere essere trasciuato.

Anche lo sviluppo di $\left(1+\frac{a}{d}\right)^{-2}$ è stato limitato generalmente ai termini quadratici, perchè $\frac{x}{d}$ puó raggiungere al massinno il valore di $1 / 3$; il termine di ter'zo gratro, patri a $4\left(\frac{x}{d}\right)^{3}$, vale percio circa $1 / 8$. Tenento presente che le variazioni relative di resistivita alparente nel passaggrio da uno stendimento all'altro raggiungono difficilmente il $10 \%$, si deduce che effettivamente i terninini di $3^{\circ}$ ordine sono piccoli exd è senz'altro 
sufficiente tenerme ronto solo per il calcolo del coefficiente della derivata prima, comes ie stato fatto.

Si ottiene cosi:

$$
\begin{gathered}
\varrho(d+x) \cdots(d)=\left\{\varrho^{\prime} x+\frac{1}{2} \varrho^{\prime \prime} x^{2}+\frac{1}{6} o^{\prime \prime \prime} x^{3} \ldots\right\} \\
\left(1+\frac{x}{d}\right)^{2} \\
\left\{1-2 \frac{x}{d}+3 \frac{x^{2}}{d^{2}}-4 \frac{x^{3}}{d^{3}} \ldots\right\}= \\
=\varrho x-\left(2 \frac{\varrho}{d}-\frac{1}{2} \varrho^{\prime \prime}\right) x^{2}+ \\
+\left(3 \frac{\varrho^{\prime}}{d^{2}}-\frac{\varrho^{\prime \prime}}{d}+\frac{1}{6} \varrho^{\prime \prime \prime}\right) x^{3}+
\end{gathered}
$$$$
\left(-1 \frac{Q^{\prime}}{d^{3}}+\frac{3}{2} \frac{\varrho^{\prime \prime}}{d^{2}}-\frac{1}{3} \frac{Q^{\prime \prime \prime}}{d}\right) x^{2}+\frac{1}{2} \varrho^{\prime \prime \prime} \frac{x^{5}}{d^{2}}
$$

Moltiplicando per d $x$, integrando e limitando tra - a e $+a$, i termini con le potenze pari si eliclono e rimane:

$$
\begin{aligned}
& \varrho_{a}-\varrho_{o}=\frac{1-y^{2}}{2 a} \mid-\frac{2}{3}\left(9 \frac{o^{\prime}}{d} \cdot-\frac{1}{2} \varrho^{\prime \prime}\right) a^{3}+ \\
& +\frac{2}{5}\left(-+\frac{o^{\prime}}{d^{3}} \div \frac{3}{2} \frac{Q^{\prime \prime}}{d^{-}}-\frac{1}{3} \frac{\varrho^{\prime \prime \prime}}{d}\right) a^{5} \mid
\end{aligned}
$$

ovvero, ponendo $a=d y$ :

$$
\begin{aligned}
& Q_{a}-o_{o}=\left(1-y^{2}\right)\left[-\left(\frac{2}{3}+\frac{4}{5} y^{2}\right) o^{\prime}+\right. \\
& \left.+\frac{1}{2}\left(\frac{1}{3}+\frac{3}{5} y^{\prime \prime}\right) a \cdot y^{\prime \prime}-\frac{1}{15} y=a z^{\prime \prime \prime}\right] \cdot y^{2} d
\end{aligned}
$$

Fel caso in cui $y=\frac{1}{3}$ (dispositivo Wenner) si ha:

$$
\begin{gathered}
l=o_{a}-\varrho_{o}=-0,07+7 d \cdot \varrho^{\prime}+ \\
-0,0198 d \cdot \varrho^{\prime \prime}-0,0007 d^{3} \varrho^{\prime \prime \prime}
\end{gathered}
$$

La correzione può essere limitata al secondo termine. I coefficienti numerici dei termini successivi, infatti sono assai piccoli; rome si vede, quello del terzo termine è circa il $4 \%$ di quello del secondo. Perciò, anche se la derivata terza ha un valore sensibile, l'influenza di tale termine diviene consistente solo a grandi distanze, ove la precisione delle misure non può essere molto elevata.

$$
\text { Consideriamo il caso di } \frac{a}{d}=\frac{1}{3} \text { come }
$$

valore massimo raggiungibile nella pratica degli stendimenti dei sondaggi elettrici ed esaminiamo perciò con la [3] il contributo di ciascuno dei due termini nella correzione totale da apportare ai valori di $\varrho$ per trasformare una curva "Schlumberger" in una "Wenner " e viceversa.

Is orvio che, quando la curvatura è nulla vi è solo da considerare il contributo del $1^{\circ}$ termine; in questo caso può essere comodo apportare alla curva, anzichè la correzione di ordinata (o), quella di ascissa $(x)$ :

$$
x_{a}-x_{o}=\frac{\varrho_{a}-\varrho_{o}}{\varrho^{\prime}}=-0,0747 d \text {. }
$$

Ricordando inoltre che i diagrammi dei sondaggi elettrici vengono disegnati sul piano logaritmico, si ha che, per i tratti rettilinei, basta spostare la curva di ma quantità costante lungo l'asse delle ascisse. Dai segni dei termini della [3] si rileva anche che, quando le curve scende, la correzione è positiva (passando dla $\varrho_{o}$ a $\varrho_{a}$ ); quando sale, la correzione è negativa, e cioè la curva "Wenner" si trova al disotto della Schlumberger: è noto, difatti, che le curve del primo tipo sono più "attenuate" di quelle del secondlo. Si nota ancora che, passando da $Q_{a}$ a $o_{o}$, la correzione per il secondo termine deve essere apportata sempre verso la parte interna della curva.

Nei tratti dove la eurvatura diviene sensibile, il secondo termine non è più trasc'urabile e sorge il problema di come calcolare $o^{\prime \prime}$ con esattezza sufficiente; difatti, se si sceglie un intervallo piccololo, gli errori di lettuma portano una imprecisione sensibile nella determinazione delle curvature: se l'intervallo è grande, non si tiene conto della forma reale della curva. Occorre una certa esperienza per la scelta dell'intervallo più opportuno.

Un criterio può essere anche quello di eseguire l'integrazione grafica limitatamente a qualche punto delle zone in cui la curvatura è più forte.

In fig. 2 è riportato un diagramma teorico per il caso di 3 strati, calcolato per il dispositivo Schlumberger (tratto continuo); i rap)- 
porti di resistività sono stati scelti in modo che lat curvatur"s, nella parte inferiore della curral, fosse assai sensibile. La curva tratteggiata è quella ottenuta apportando, ai valori di o letti sul diagramma, la correzione calcolata con lit [3] limitata al 20 termine. ronsiderato uguale a zero. Difatti, anmentando la distanza tra gli elettrodi, la tensione misurata alle sonde si abbassa, fino a non poter essere più determinata con la precisione occorrente. Per nom spostare le sonde troppo frequentemente, la misura, per lo

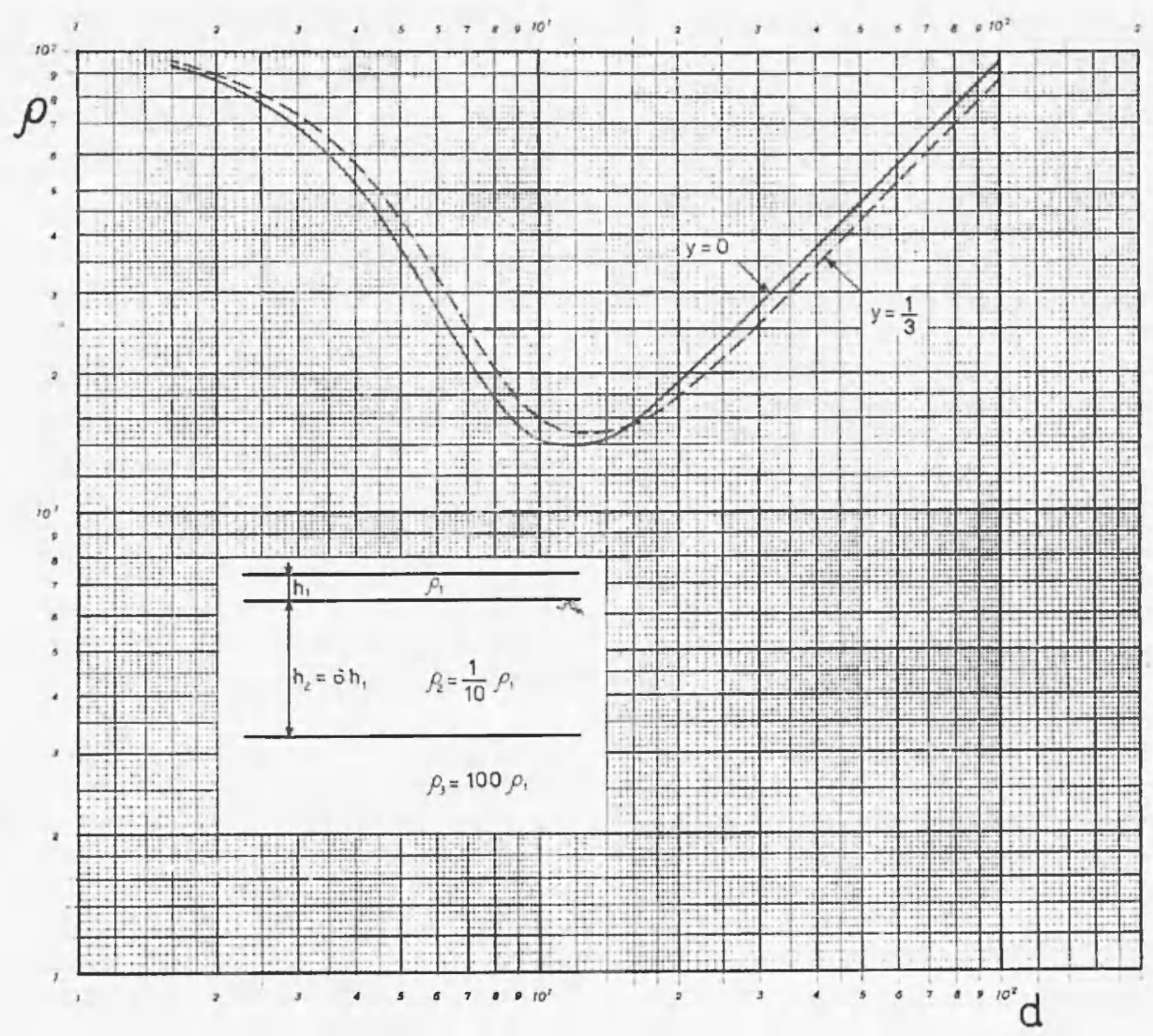

Fig. 2

Alcuni controlli eseguiti integrando graficamente la [1], hamno dimostrato che l'approssimazione ragguiunta con la [3] è del tutto soddisfacente.

Il procedimento descritto può essere aplicato utilmente, oltre che per trasformare le curve teoriche, anche per eliminare delle deformazioni talvolta piuttosto sensibili, negli stessi diagrammi sperimentali di campagna. Come è noto, impiegando il dispositivo Schlumberger, il rapporto $y=\frac{a}{d}$ non rimane costante e non può essere sempre stesso $d$, viene ripetuta con a notevolmente più grande. In tal modo $y$ può raggiungere il valore di $\frac{1}{5}$ e, talora, anche di $\frac{1}{3}$. In questi punti di "riattaceco n i valori misurati di $\varrho$ con i due diversi a possono divergere sensibilmente. Questo fatto ha due cause: la prima è the le condizioni superticiali nell'interno delle sonde sono variate; la seconda è che ci si discosta dall'ipotesi $y=0$. Lat differenza delle condizioni superficiali si corregge assai semplicemente, traslando parallelamente i vari tratti di curva in modo da 
portarli a coincidere. Lo scalto causato dalla variaziono di y, invere, si puó correggeres con la [2], calcolando i coefficienti jere valli $y$. In preatica bastera determinare le correzioni solo per i valori piil grandi dal rapporto, poichè già per $y=\frac{1}{8}$ il coefficiente del primo termine diviene $1 / 7$ di quello per $y=\frac{1}{3}$ e la correzione è perciò trascumabile.

Nolla maggiomanza dei casi, è sufficiente determinare la differenza di o nel punto di riattaceo e raceordare la curva corretta con il punto del diagramma osservato nel quale $y$ is sufficientemente pircolo.

Quando i $K$ a dol diagramma sono celevati e il "riattareco "arviene proprio nei pressi dellat massima curvatura, cioè y è valriabile quando og è sensibile, ocroore procedere con maggiore cautela per' non deformare la curva Si nota peró dalla [2] che, a parita di $d$, la correzione dovuta al secondo termine è sensibilmeste proporzionale ad $y^{2}$.

Un criterio che garantisce ma arematezza notevole è quello di calcolare per mezzo delle [3], solo per il tratto curvo, i diagrammi teorici di Wemmer, e di rlisegnarli sulle curve interpretative. Confrontanto questo tratto di diagramma teorico con quello osserrato, è più agevole eseguire il racordo tra il punto di "riattareo e quello dove la correzione diviene trascumabile. La correzione ai valori misuluti viene così derlotta direttamente dalle curre; essa dere essere eventualmente ridotta, se $a<a_{0}=\frac{1}{3} d$, nel rapporto $\left(\frac{a}{a_{o}}\right)^{2}$.

\section{RIASSTNO}

Tiene proposto un metodo approssimato wer trasformare $i$ diagrammi di resistiviti ottenuti con differenti dispositivi elettrodici, in quelli ricavati seguendo lo schema Schlumberger, o viceversa. Il criterio esposto, di applicazione assai rapida, si presta anche a risolvere il problema, che sorge durante le misure in campagma, di raccordare $i$ vari tratti di curva osservata quando cambia la distanza fra le sonde del potenziale.

\section{ABSTRACT}

A rapid method is proposed to transfer the observed resistivity curves obtained with any electrode configuration into schlumberger diagrams. Such procedure is of practical. application even to solve the field problem of fitting the different sections of the diagrams, uhen the distance between the voltage eiectrodes changes.

\section{BIBIJIOGRAFIA}

(1) Belluigi, A., Su una "exala quaestio " di gerarchia nei dispositivi dei sondaggi eletirici. "Geofisica pura e Applicata ", XXIV, 1956.

(2) - Abaques de sondage éléctrique par la Compagnie Gienerale de Géophysique. "Geophysical Prospecting ", 1955, suppl. 3.

$\left.{ }^{3}\right)$ Alyaxo, I.. Studi sulla interpretazione dei sondaggi elettrici verticali. "Rivista di Geofisica 1 pplicata ", 2, 1951. 